\title{
The Equipment Target Price Adjusting Model Based on Uncontrollable Technical Risk
}

\author{
SUN Sheng-xiang ${ }^{1, a}$, HAN Shuang ${ }^{2, b,{ }^{*}}$ and XIE Li ${ }^{3, c}$ \\ ${ }^{1,2,3}$ Department of Equipment Economics and Management, Naval University of Engineering, \\ Wuhan, Hubei Province, China \\ a575986943@qq.com, b331262279@qq.com, ‘839875073@qq.com \\ ${ }^{*}$ Corresponding author
}

Keywords: equipment target price adjusting, incentive pricing, uncontrollable technical risk, cost control

\begin{abstract}
In view of the uncontrollable technical risk resulted in the actual cost is far more than the target price in the process of equipment development, the mode of existing incentive - constrained pricing cannot play an effective role, the equipment suppliers lose enthusiasm for cost control. Equipment target price adjusting model under a single uncontrollable technical risk is given. For the difference of cost overrun and saving caused by uncontrollable technical risk, the cost allocation coefficient and the cost sharing coefficient are given based on the asymmetric incentive theory. On this basis, the equipment target price adjusting models under two or more uncontrollable technical risks are developing, which can provide decision reference for the precise motivation of the actual equipment pricing. Finally, an example is used to verify the feasibility and effectiveness of model.
\end{abstract}

\section{基于不可控技术风险的装备目标价格调整模型}

\author{
孙胜祥 ${ }^{1, a}$, 韩霜 $2, b,{ }^{*}$, 谢力 ${ }^{3, c}$ \\ $1,2,3$ 海军工程大学管理工程与装备经济系, 武汉, 湖北, 中国 \\ a575986943@qq.com, b331262279@qq.com, '839875073@qq.com \\ ”通讯作者
}

关键词：装备目标价格调整；激励定价；不可控技术风险；成本控制

中文摘要. 针对在装备研发过程中不可控技术风险发生导致实际成本远超目标价格时, 现有 的激励约束定价模式往往无法发挥激励作用，承制单位失去成本控制的动力的问题，构建了 单项不可控技术风险下的装备目标价格调整模型。结合不可控技术风险导致成本超支和成本 节约时不同的特点, 基于不对称激励理论, 分别设置了军方不可控技术风险成本分推系数及 分享系数; 在此基础上，构建了两项和多项不可控技术风险下的装备目标价格调整模型，为 装备实际定价工作的精准激励提供决策参考。最后, 通过案例验证了模型的可行性和有效性。

\section{1. 引言}

随着社会主义市场经济体制的逐步完善，装备价格工作改革持续推进。根据装备购置目 标价格形成机制、过程成本监控机制和激励约束机制 ${ }^{[1]}$, 装备的目标价格是贯穿其中的核心 因素, 将直接影响购置价格的合理性以及激励效果的发挥。由于装备研发过程是一项科技含 
量高、复杂的系统工程, 而目标价格在论证阶段进行确定, 此时装备面临较大技术风险, 技 术状态存在不确定性, 使得最初的装备目标价格只能是初略的测算, 可能与实际情况存在巨 大偏差 ${ }^{[2]}$ 。在所发生的技术风险中，有一部分是承制单位无法控制的，但由此造成的巨额损 失仍需承制单位自行承担, 即使之后承制单位对成本进行控制往往也无济于事, 所以承制单 位通常在风险发生后便丧失了成本控制的动力, 这严重影响了军费的使用效益。因此, 如何 在不可控技术风险发生的情况下, 优化调整目标价格, 从而对承制单位实施精准激励, 以提 高其持续进行成本控制的动力具有重要的现实意义。

目前, 相关学者对激励承制单位成本控制问题进行了一定的研究，范道津 ${ }^{[3]}$ 运用期望-效 用函数及帕累托最优理论分析成本激励系数的确定问题，建立了相应的计算模型。訾书宇 ${ }^{[4]}$ 基于利润最大化原则, 提出了目标价格合同的三参数优化设计方法。以上学者从不同的角度 对激励因子的设计进行了研究, 但仅考虑了激励力度的问题, 未考虑激励对象的基本参照值 一目标价格是否准确。钟强晖 ${ }^{[5]}$ 对目标价格进行了研究, 结合装备研制的特点, 从理论层 面给出了装备不同研制阶段的目标价格调控工作内容，但未提出具体的价格调整方法。

综合上述分析，本文在对不可控技术风险分析的基础上，拟通过调整目标价格的方法， 使军厂双方以合理的比例共同承担不可控技术风险所造成的影响。期望调整后的目标价格, 一方面能为装备激励约束定价提供依据, 发挥精准激励的效果; 另一方面能激励承制单位进 行持续的成本控制，提高军费的使用效益。

\section{2. 装备研发中的不可控技术风险分析}

在装备研发过程中往往伴随着技术风险的发生，即由于各种技术因素及其影响作用的不 确定性, 导致装备的性能、费用、进度与预期目标发生偏离, 对装备的研发产生影响 ${ }^{[6]}$, 具 体体现为设计风险、试验风险、生产风险、管理风险 ${ }^{[7]}$ 。对承制单位而言，有些技术风险通 过自身的努力可以避免, 但有些是无法控制的。根据承制单位对于技术风险的控制能力, 可 以将其分为可控技术风险和不可控技术风险两种。其中，不可控技术风险是指在应对研发技 术复杂性与不确定性上, 并非完全受承制单位努力程度的控制, 即使其付出了巨大的努力有 时也会超出承制单位的承受控制能力。

装备研发过程中的不可控技术风险具有不可合理预见、不能避免或和不可控等特点，根 据不可控技术风险的发生对成本所造成的影响，又可将其分为成本超支型和成本节约型。其 中, 成本超支型不可控技术风险较为常见, 为控制或解决其发生所带来的问题需要增加成本 的投入，如在既定的资源和时间约束下，装备研发中采用的某项或几项技术不能较好地完成 既定的任务需求, 需反复试验以确保质量, 从而造成成本的大幅增加。而成本节约型不可控 技术风险的发生使成本减少, 如在研发过程中, 出现了价格低效果好的新材料、新工艺, 采 用其替代原设计中的材料及工艺来实现预期功能，使总成本降低。

由于不可控技术风险的发生与承制单位自身的努力程度无关，因此在使用激励约束定价 时, 如果不可控技术风险导致成本超支，军方应分摊大部分的超支额，调高目标价格; 如果 不可控技术风险导致成本节约, 军方可视情分享一部分节约额, 承制单位保留大部分节约额, 适当调低目标价格。这样才能最大程度地发挥激励效果，达到精准激励的目的。

\section{3. 单项不可控技术风险下的调整模型}

\section{1 模型构建}

为简化问题，对本模型提出以下假设:

（1）装备研制过程中，仅考虑不可控技术风险发生带来的成本变化，而政策、自然、市 场等其他不可控风险则能通过相应的保险机制进行管理。

(2) 军方能够掌握不可控技术风险发生时实际成本的变化。 
（3）军方属于风险规避, 对成本超支风险是厌恶的, 在目标价格确定过程中通常会设定 价格上限, 即不得超过目标成本的百分比, 当最终价格超过军方最高限价时, 项目中止。

装备目标价格由目标成本和目标利润两部分组成，公式表示为:

$$
P_{t}=C_{t}+f
$$

式中, $P_{t}$ 为初始目标价格; $C_{t}$ 为初始目标成本，由制造成本和期间费用确定; $f$ 为目标 利润，由目标成本与固定利润率5\%共同决定，确定后不再变化。

按照不可控技术风险发生时调整装备目标价格的思路，装备的目标成本由两部分组成:

(1) 可控成本 $C_{y}$, 具体是指承制单位通过努力可以控制的成本，大小与努力程度有关。 $C_{y}$ 是承制单位努力程度的函数 $C_{y}=C_{y}(e)$, 努力可以是正向努力, 则该部分成本减少, 也可以 是负向努力, 该部分成本增加。函数如图1所示:

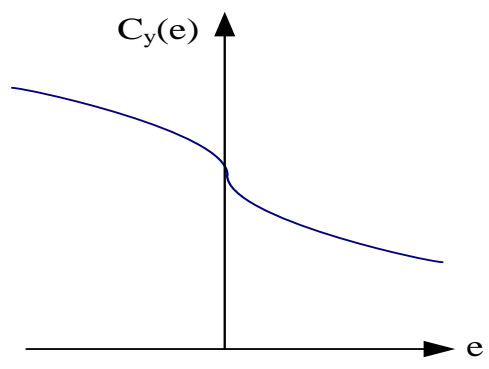

图1 努力程度函数

（2）不可控技术风险成本 $C_{x}$ ，即不可控技术风险的发生导致的成本变化。根据上文分 析, 成本超支型不可控技术风险发生时, $C_{x}>0$; 成本节约型不可控技术风险发生时, $C_{x}<0$ 。 供需双方在研制立项论证时协商确定目标价格，此时的目标成本主要由可控成本 $C_{y}$ 的协 商值确定，因此根据式（1），初始目标价格为:

$$
P_{t}=C_{y} \times(1+5 \%)
$$

当单项不可控技术风险发生时，将产生不可控技术风险成本 $C_{x}$ 。此时对目标价格进行调 整, 使军方与承制单位共同分摊（分享） $C_{x}$, 假设 $\alpha$ 为不可控技术风险成本共享系数, 即军 方对不可控技术风险导致的成本变化的分摊（分享）比例。则调整后的目标价格为:

$$
P_{t}^{\prime}=C_{y} \times(1+5 \%)+C_{x} \times \alpha
$$

\section{2 不可控技术风险成本共享系数的求解}

单项不可控技术风险的发生对成本产生两种影响，对外表现为超支和节约。根据成本超 支与成本节约这两种情况，基于不对称激励思想，将 $\alpha$ 分为不可控技术风险成本分推系数与 成本分享系数, 分别求解。

\subsection{1 不可控技术风险成本分摊系数}

不可控技术风险导致成本超支时，军方为保护承制单位，避免其承担全部损失，对超支 部分进行分推。考虑到成本超支比例反映了项目的不可控技术风险程度, 军方对超支部分的 分摊比例应随超支比例动态变化。具体而言, 当超支比例较小时, 代表不可控技术风险较低, 此时承制单位有能力承担部分损失; 超支比例较大时则风险较高, 为保证承制单位研发顺利 进行, 军方承担超支部分的份额应增加。不可控技术风险成本分摊系数 $\alpha_{a}$ 随不可控技术风险 成本超支比例 $r_{a}$ 的变化关系，如图2所示:

不妨设:

$$
\alpha(r)=(m-1) e+1
$$

式中 $m$ 可以看作是军方不可控技术风险成本的基准分推系数, 可根据承制单位风险管理 的能力以及项目的技术成熟度等具体确定, $m$ 为常数, $0<m<1$ 。 


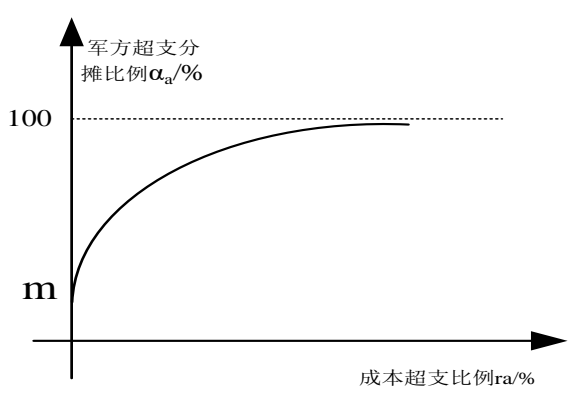

图2 成本超支比例与军方分推比例关系

\subsection{2 不可控技术风险成本分享系数}

不可控技术风险导致成本节约时，为鼓励承制单位积极主动追求成本节约，对于节约额 军方应以合理比例与承制单位共同分享。当成本节约比例较低时, 将大部分节约额给予承制 单位, 以提高其生产积极性; 当节约比例较高时, 军方可适当分享部分节约额。不可控技术 风险成本分享系数 $\alpha_{b}$ 随不可控技术风险成本节约比例 $r_{b}$ 的变化关系，如图3所示:

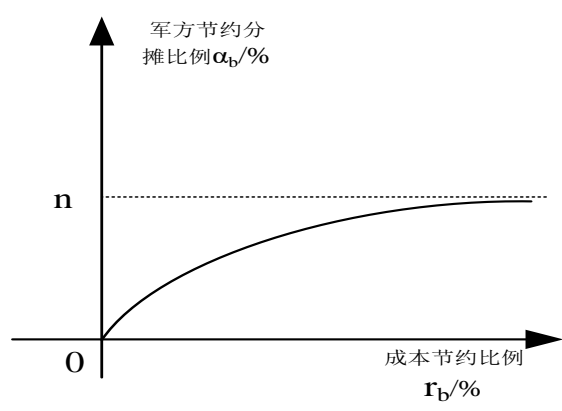

图3 成本节约比例与军方分摊比例关系

不妨设:

$$
\alpha_{b}\left(r_{b}\right)=n\left(1-e^{-r_{b}}\right)
$$

式中 $n$ 可以看作是军方不可控技术风险成本的基准分享系数，可根据承制单位风险管理 的能力以及项目的技术成熟度等具体确定, $n$ 为常数, $0<n<1$ 。

综上，单项不可控技术风险发生时，调整后的目标价格为:

$$
\begin{aligned}
& P_{t}^{\prime}=C_{y} \times(1+5 \%)+C_{x} \times \alpha \\
& \text { s.t. }\left\{\begin{array}{l}
\alpha=\left\{\begin{array}{l}
(m-1) e^{-r}+1\left(C_{x}>0\right) \\
n\left(1-e^{-r}\right)\left(C_{x}<0\right)
\end{array}\right. \\
0<m<1, \quad 0<n<1 \\
r=\left|\frac{C_{x}}{C_{y}}\right|
\end{array}\right.
\end{aligned}
$$

\section{4. 两项不可控技术风险下的调整模型}

装备研发过程中所面临的不可控技术风险往往并非仅有一项，越是先进的装备，不确定 因素往往越多。这些不可控技术风险对于实际成本的影响有的是非关联的（独立起作用）, 有的是关联的（相互影响共同产生作用）。下面针对两项非关联不可控技术风险和关联技术 风险下的调整模型进行探讨。 


\section{1 两项非关联的不可控技术风险下的调整模型}

在装备研制过程中，当两项非关联不可控技术风险发生时，由于各项技术风险对装备实 际成本的影响相互独立, 因此, 可以逐项按照单项不可控技术风险的调整模型重复计算、依 次调整。

\section{2 两项关联的不可控技术风险下的调整模型}

在研制过程中两项相互影响的不可控技术风险发生时, 不可控风险1的发生将对后期不可 控风险 2 发生时实际成本的变化产生一定的影响。

调整后的目标成本为:

$$
C_{t}^{\prime}=C_{y}+C_{x_{1}} \times \alpha\left(r_{1}\right)+C_{x_{2}} \times\left[\alpha\left(r_{2}\right)+\theta_{2 \mid 1}\right]
$$

式中, $C_{x_{1}} 、 C_{x_{2}}$ 分别为不可控技术风险 $1 、 2$ 发生带来的成本变化; $\alpha\left(r_{1}\right) 、 \alpha\left(r_{2}\right)$ 分别为军 方对不可控技术风险 $1 、 2$ 的成本分推比例; $\theta_{2 \mid 1}$ 为不可控风险 1 的发生对不可控风险 2 发生时实 际成本变化的影响因子, 服从均值为 0 , 方差为 $\delta_{1}^{2}$ 的正态分布。

两项关联的不可控技术风险发生时, 调整后的目标价格为:

$$
\begin{aligned}
& P_{t}^{\prime}=C_{y} \times(1+5 \%)+C_{x_{1}} \times \alpha\left(r_{1}\right)+C_{x_{2}} \times\left[\alpha\left(r_{2}\right)+\theta_{2 \mid 1}\right] \\
& \text { s.t. }\left\{\begin{array}{l}
\alpha(r)=\left\{\begin{array}{l}
(m-1) e^{-r}+1\left(C_{x}>0\right) \\
n\left(1-e^{-r}\right)\left(C_{x}<0\right)
\end{array}\right. \\
0<m<1, \quad 0<n<1 \\
r=\left|\frac{C_{x}}{C_{y}}\right|
\end{array}\right.
\end{aligned}
$$

\section{5. 多项不可控技术风险下的调整模型}

当多项非关联不可控技术风险在装备研制过程中发生时, 由于各项风险对成本的影响相 互独立, 因此, 可以逐项按照单项不可控技术风险的调整模型重复计算、依次调整。下面主 要对多项关联不可控技术风险下的装备目标价格调整模型进行讨论。

当有 $k$ 项 $(k>2)$ 具有关联的不可控技术风险发生, 共同影响装备实际成本时, 对目标 成本进行调整:

$$
\begin{aligned}
C_{t}^{\prime} & =C_{y}+C_{x_{1}} \times \alpha\left(r_{1}\right)+C_{x_{2}} \times\left[\alpha\left(r_{2}\right)+\theta_{2 \mid 1}\right]+C_{x_{3}} \times\left[\alpha\left(r_{3}\right)+\theta_{3 \mid 1}+\theta_{3 \mid 2}\right]+\ldots+C_{x_{k}} \times\left[\alpha\left(r_{k}\right)+\theta_{k \mid 1}+\ldots+\theta_{k \mid k-1}\right] \\
& =C_{y}+\sum_{i=1}^{k} C_{x_{i}} \times \alpha\left(r_{i}\right)+\sum_{j=2}^{k} \sum_{i=1}^{k-1} C_{x_{j}} \times \theta_{j \mid i}
\end{aligned}
$$

式中, $C_{x_{i}}$ 为不可控技术风险 $i$ 发生带来的成本变化; $\alpha\left(r_{i}\right)$ 为军方对不可控技术风险 $\mathrm{i}$ 的成 本分摊比例; $\theta_{j i}$ 为不可控风险 $i$ 的发生对不可控风险 $j$ 发生时实际成本变化的影响因子。

多项关联的不可控技术风险发生时, 调整后的目标价格为:

$$
\begin{gathered}
P_{t}^{\prime}=C_{y} \times(1+5 \%)+\sum_{i=1}^{k} C_{x_{i}} \times \alpha\left(r_{i}\right)+\sum_{j=2}^{k} \sum_{i=1}^{k-1} C_{x_{j}} \times \theta_{j i i} \\
\text { s.t. }\left\{\begin{array}{l}
\alpha\left(r_{i}\right)=\left\{\begin{array}{l}
(m-1) e^{-r_{i}}+1\left(C_{x_{i}}>0\right) \\
n\left(1-e^{-r_{i}}\right)\left(C_{x_{i}}<0\right)
\end{array}\right. \\
0<m<1, \quad 0<n<1 \\
r_{i}=\left|\frac{C_{x_{i}}}{C_{y}}\right|
\end{array}\right.
\end{gathered}
$$




\section{6. 案例分析}

\section{1 背景介绍}

某装备采购部门需采购某型舰船装备，经过立项论证与方案设计，军方与承制单位协商 确定目标成本为 20.66 亿元（目标价格为21.69亿元），最高限价为 24.36 亿元。装备采购部门 持续跟踪控制目标价格的实现情况，在技术方案发生重大变化等技术风险发生时，组织专家 组开展专项技术分析与成本评估。

在工程研制与设计定型过程中发现，完成动力系统原定指标的技术难度较大，为实现预 期目标需在动力系统中增加设备 $\mathrm{A}$, 成本增加共计 0.533 亿元。另外, 经某研究所多年努力, 在推进设备B的安装技术方面实现重大突破，承制单位通过引进该项技术使成本节约共计 0.33 亿元。但由于设备A的增加, 需进行多次试验以确保设备A、B的配合效果, 成本增加共 0.242 亿元。假设该型舰船的研发过程中, 除上述成本发生变化外, 其他均按照原价格设计方案实 施, 则最终的实际成本为 21.105 亿元。

\section{2 模型运用及结果分析}

若采用现有的定价方法，则合同最终目标成本仍为 20.66 亿元，因此实际成本超支4450万 元，承制单位将承受巨大损失，即使后续进行成本控制能使成本一定程度减小，但想最终获 利可能性也很小。显然在此定价方法下，不可控技术风险发生后承制单位失去了成本控制的 动力。

若采用基于不可控技术风险的装备目标价格调整模型，在不可控技术风险发生时，允许 对目标成本进行调节。专家组结合历史成本数据及承制单位研发实际, 确定军方的基准技术 风险成本分摊系数 $m=63.5 \%$, 基准技术风险成本分享系数 $n=41.2 \%$, 同时确定两项风险间的影 响因子 $\theta_{2 \mid 1}=-0.584$ 。根据式（4）、（5），分别得到技术风险成本补偿系数:

$$
\begin{aligned}
& \alpha_{1}=(m-1) e^{-r_{a}}+1=(0.635-1) e^{-\frac{0.533}{20.66}}+1=64.43 \% \\
& \alpha_{2}=n\left(1-e^{-r_{b}}\right)=0.412 \times\left(1-e^{-\frac{0.33}{20.66}}\right)=0.65 \% \\
& \text { 风险 } 1 \text { 发生后, 根据式 }(6), \text { 可得到调整后的目标成本为: } \\
& C_{t}^{\prime}=C_{y}+C_{x} \times\left[(m-1) e^{-r_{a}}+1\right]=20.66+0.533 \times 64.43 \%=21.00 \text { 万元 }
\end{aligned}
$$

目标成本为 21.00 万元, 则实际成本超支 1050 万元, 相比于之前亏损的 4450 万元, 承制单 位后期继续加强成本控制, 最终实现获利的可能性大幅提升, 这在一定程度上提高了承制单 位成本控制的动力。

当不可控技术风险 2 发生后，根据式（7），可得该型舰船装备调整后的目标成本为:

$C_{t}^{\prime}=C_{y}+C_{x_{1}} \times \alpha\left(r_{1}\right)+C_{x_{2}} \times\left[\alpha\left(r_{2}\right)+\theta_{2 \mid 1}\right]=20.66+0.533 \times 64.43 \%-0.33 \times(0.65 \%-0.584)=21.19$ 万元

则实际成本节约了 850 万元。也就是说, 虽然风险发生导致成本出现大幅超支, 但通过军 方对损失的分推, 再加上承制单位引进新技术使成本节约, 最终承制单位除获得目标利润外, 还将因成本节约获得奖励。因而, 基于不可控技术风险的装备价格调整模型可以有效地激励 承制单位进行持续的成本控制，提高军费的使用效益。

\section{7. 结束语}

基于不可控技术风险的装备目标价格调整模型, 旨在得到合理的目标价格, 以实现精准 激励承制单位的目的。

针对装备研发过程中，承制单位需自行承担与自身努力程度无关的不可控技术风险的问 题, 提出了在风险发生后对目标价格进行调整, 由军方与承制单位以合适的比例共同分推/分 享风险所造成的损失/收益。首先，通过分析不可控技术风险对实际成本产生的影响，将不可 控技术风险分为成本超支型与成本节约型。其次, 基于不对称激励的理论, 设置了军方技术 
风险成本分推系数及成本分享系数, 并分别构建了成本超支/节约型的单项不可控技术风险下 的调整模型。再次, 以单项不可控技术风险价格调整模型为基础, 进一步考虑两项及多项不 可控技术风险间的相互关系及影响，构建了两项和多项不可控技术风险下的调整模型。最后， 以具体案例说明了这种目标价格调整模型的合理性和有效性。

由于本文的研究建立在风险导致的成本变化值已知的假设条件基础上, 后续还需要继续 开展成本值确定流程及方法的研究, 使成果更加接近装备定价实际。在不可控风险发生时对 目标价格进行动态调整, 将其产生的影响从目标价格中分离出来的思路也可为承制单位成本 控制工作提供参考，不断提高成本管理水平。

\section{致谢}

本文为国家社科基金面上项目（18BGL287）的阶段性成果之一。

\section{References}

[1] S. Y. Sun, L Xie, and M. C. Lin, Equipment pricing, Beijing: National Defend Industry Press, pp. 300-303, 2018.

[2] S. Y. Zi, M. C. Lin, and L Xie, Incentive Price Contract Mode of Military Equipment Based on Cost Estimation Risk, Journal of Academy of Armored Force Engineering, vol. 32, pp. 33-37, 2018.

[3] D. J. Fan and Y. L. Du, Practice of Enterprise-based Agent-building System, Tianjin: Tianjin University Press, pp. 67-69, 2010.

[4] S. Y. ZI, R. X. WEI, and H. Jin, Optimal design method of target price contract, Journal of Naval University of Engineering, vol. 2, pp. 49-53, 2014.

[5] Q. H. Zhong, M. C. Lin, and G. J. Ping, Study on Target Price Adjusting and Controlling in Equipment Developing Process, Science and Technology Management Research, vol. 14, pp. 208-215, 2013.

[6] Q. Zhang, Z. P. Fan, and D. H. Pan, A Ranking Approach for Interval Numbers in Uncertain Multiple Attribute Decision Making Problems, Systems Engineering-Theory \& Practice, vol.19, pp. 129-133, 1999.

[7] Z. Xu, Y. C. Feng, and D. W. Lu, Appraisement model of technical risk for weapon system development, Systems Engineering and Electronics, vol. 27, pp. 1123-1127, 2005. 